\title{
Multidrug resistance protein P-gp interaction with nanoparticles (fullerenes and carbon nanotube) to assess their drug delivery potential: a theoretical molecular docking study
}

\section{Sergey Shityakov* and Carola Förster}

Department of Anesthesiology and Critical Care,

University of Würzburg,

97080 Würzburg, Germany

Email: E_Shityako_S@klinik.uni-wuerzburg.de

Email: Foerster_C@klinik.uni-wuerzburg.de

*Corresponding author

\begin{abstract}
P-glycoprotein (P-gp)-mediated efflux system plays an important role to maintain chemical balance in mammalian cells for endogenous and exogenous chemical compounds. However, despite the extensive characterisation of P-gp potential interaction with drug-like molecules, the interaction of carbon nanoparticles with this type of protein molecule is poorly understood. Thus, carbon nanoparticles were analysed, such as buckminsterfullerenes $\left(\mathrm{C}_{20}, \mathrm{C}_{60}, \mathrm{C}_{70}\right)$, capped armchair single-walled carbon nanotube (SWCNT or $\mathrm{C}_{168}$ ), and P-gp interactions using different molecular docking techniques, such as gradient optimisation algorithm (ADVina), Lamarckian genetic algorithm (FastDock), and shape-based approach (PatchDock) to estimate the binding affinities between these structures. The theoretical results represented in this work show that fullerenes might be P-gp binders because of low levels of Gibbs free energy of binding $(\Delta \mathrm{G})$ and potential of mean force (PMF) values. Furthermore, the SWCNT binding is energetically unfavourable, leading to a total decrease in binding affinity by elevation of the residual area $\left(\mathrm{A}_{\text {res }}\right)$, which also affects the $\pi-\pi$ stacking mechanisms. Further, the obtained data could potentially call experimental studies using carbon nanostructures, such as SWCNT for development of drug delivery vehicles, to administer and assess drug-like chemical compounds to the target cells since organisms probably did not develop molecular sensing elements to detect these types of carbon molecules.
\end{abstract}

Keywords: P-glycoprotein; carbon nanoparticles; fullerenes; single-walled carbon nanotube; molecular docking; Gibbs free energy of binding; potential of mean force; $\pi-\pi$ stacking.

Reference to this paper should be made as follows: Shityakov, S. and Förster, C. (2013) 'Multidrug resistance protein P-gp interaction with nanoparticles (fullerenes and carbon nanotube) to assess their drug delivery potential: a theoretical molecular docking study', Int. J. Computational Biology and Drug Design, Vol. 6, No. 4, pp.343-357.

Biographical notes: Sergey Shityakov is currently a Postdoctoral Fellow in the Department of Anesthesiology and Critical Care at Würzburg Hospital University. He received an MD in Immunology and Infectious Diseases from Novgorod State University School of Medicine in Russia, and a $\mathrm{PhD}$ in Molecular Biology from Würzburg University in Germany. His research interests include blood-brain barrier research, molecular docking, and 
molecular dynamics simulation of different biological systems, such as brain transporter proteins and carbon nanostructures. His work in these areas has been published in various peer-reviewed journals and presented at scientific conferences.

Carola Förster is a Professor in the Department of Anesthesiology and Critical Care at Würzburg Hospital University. She received an MS in Biochemistry and a $\mathrm{PhD}$ in Molecular Biology from Hannover University in Germany. Her main research topics have included the role of the blood-brain barrier in inflammatory diseases and brain injury, effects of maternal inflammation on the blood-brain barrier integrity in fetal brain development, stem cell therapy, and regenerative medicine after brain injury, characterisation and pharmacological modulation of selected transporter proteins in the brain. She has participated in numerous IZKF, BMBF, and DFG projects.

\section{Introduction}

On the verge of bionanotechnology and supramolecular chemistry, the potential nanosystems, such as buckminsterfullerenes and carbon nanotubes, are expected to profit on every economic and scientific area, including pharmacology and medicine for diagnosis, bioimaging, and drug delivery (Xia et al., 2009; Johnston et al., 2010). These nanosystems are characterised by high molecular surface area and have advantages; they can be easily chemically modified to adjust their pharmacokinetics/pharmacodynamics penetration rate, various administration routs, robustness of transportation, and low toxicity, at least for the functionalised versions of carbon nanoparticles (Cataldo and Da Ros, 2008). Nevertheless, some cellular toxicity is believed to be associated with the aggregation of nanoparticles in the lipid membranes posing a threat to functional integrity of the target cells (Foley et al., 2002; Sayes et al., 2005; Porter et al., 2006).

A growing family of synthetic carbon allotropes, especially fullerene $\mathrm{C}_{60}$ and singlewalled carbon nanotubes (SWCNT), has many potential applications depending on their free radical scavenging and antioxidant chemistry to diminish free radicals, inhibit viral proteases, and identify protein fingerprints for biomarker discovery and molecular sensing (Bonn et al., 2007). Unfortunately, different xenobiotic substances, such as antibiotics and anti-cancer agents, may be expelled from living cells via several mechanisms that cause an intrinsic and acquired multidrug resistance (MDR). Among them, the efflux mechanism mediated by P-glycoprotein (P-gp) transporter, a member of $\mathrm{ABC}$ (ATP-binding cassette) superfamily, plays a major role in removing drugs out of cells and decreases their pharmacological effects (Gottesman and Fojo, 2002; AlibertFranco et al., 2009).

To overcome the MDR, a great diversity of P-gp inhibitors was produced as supplements to conventional chemotherapy (Koizumi et al., 1995; Mazel et al., 2001). However, despite the extensive analysis of P-gp interaction with drug-like molecules, the effects of buckminsterfullerenes and SWCNTs on this transporter were scarcely reported.

It was recently shown by $\mathrm{Xu}$ and coworkers using in vitro experimental techniques, combined with molecular dynamics simulations, that fullerenes were abundantly accumulated in the K562S and K562R cells probably due to the absence of P-gp efflux (Xu et al., 2012). The theoretical simulations further confirmed that $\mathrm{C}_{60}$ barely induces P- 
gp conformational shift; thus, no compelling evidence was found to claim the $\mathrm{C}_{60}$ structure as a substrate or inhibitor for P-glycoprotein. In another study, the functionalised nanoparticles, such as poly(ethylene glycol)-conjugated multi-walled carbon nanotubes, were found to be efficient drug carriers for overcoming MDR induced by the overexpression of P-gp on cellular membrane (Cheng et al., 2011). Additionally, the P-gp antibody-coupled water-soluble SWCNTs loaded with doxorubicin were synthesised for challenging the MDR of human leukemia cells and the effective loading and controllable release of the drug substances (Li et al., 2010). In fact, these functionalised nanotubes have been already widely utilised to transport different types of chemical substances including peptides, amino acids, proteins, nucleic acids, and other molecules in living cells (Pantarotto et al., 2003; Pantarotto et al., 2004; Pantarotto et al., 2004; Singh et al., 2005; Kam et al., 2005; Liu et al., 2007). Characterising P-gp binding sites of fullerenes and SWCNTs is an important step toward a better understanding of mechanisms by which nanoparticles are interacting with a typical member of the $\mathrm{ABC}$ superfamily of active transporters.

Encouraged by these experimentally derived data, we generated molecular docking models of fullerenes and SWCNT to explore the interaction of four carbon allotropes with P-gp, including three types of buckminsterfullerenes $\left(\mathrm{C}_{20}, \mathrm{C}_{60}, \mathrm{C}_{70}\right)$ and one type of capped SWCNT $\left(\mathrm{C}_{168}\right)$ closed at both sites by fullerene-like hemispheres. We believe that our approach may help to answer these questions: (1) whether there are some prerequisites for P-gp substrate or inhibitor specificity for nanoparticles of interest, and (2) what are the possible mechanisms of their interaction? To our knowledge, the current study is the first to report on the ability of P-gp to interact with lipophilic lattices of analysed nanostructures by implementing different molecular docking algorithms and scoring functions together with experimentally resolved P-gp crystallographic model to achieve more precisely determined predicting results.

\section{Computational methods}

The electron diffraction holo-structure of mouse P-glycoprotein (3G60) at $3.8 \AA$ resolution with a cyclic-ris-(R)-valineselenazole (QZ59-RRR) molecule was taken from the Protein Data Bank as a PDB file (Aller et al., 2009). Prior to molecular docking, the ligand was extracted, and the P-gp molecule was refined using FG-MD server (Zhang et al., 2011). Atomic-level protein refinement was accomplished via identification of spatial restrains from the analogous fragments to reshape the energy landscape funnel using a molecular dynamics-based algorithm (Zhang et al., 2011). The VADAR (Volume, Area, Dihedral Angle Reporter) server, which is an improved version of the PROCHECK software, was used for stereochemical validation of the P-gp molecule quality by analysing residue-by-residue, overall structure geometry and checking the $\Phi-\Psi$ bond angles in a Ramachandran plot (Willard et al., 2003). Altogether, observed statistics showed that $70 \%(23 \%)$ of all residues were in core (allowed) regions before and $82 \%$ $(12 \%)$ after the MD refinement. The expected values for the comparison were $90 \%$ and $7 \%$ for the same regions obtained from the literature elsewhere (Chiche et al., 1990; Morris et al., 1992).

Buckminster lower- $\left(\mathrm{C}_{20}\right)$ and higher-order $\left(\mathrm{C}_{60}, \mathrm{C}_{70}\right)$ fullerenes (buckyballs) were extracted from the Wellesley college chemical database. Capped armchair SWCNT $(\mathrm{n}=\mathrm{m})$ with $15.8 \AA$ in length and $8.1 \AA$ in diameter was constructed by the 
NanoEnginner-1 software (Nanorex, Inc. Bloomfield Hills, MI) with well-defined spherical tips. Different molecular docking programs, including AutoDock Vina (ADVina), Scigress FastDock (Fujitsu Scientific Software, Tokyo, Japan) and PatchDock were implemented to analyse nanoparticle interactions with the P-gp molecule (Schneidman-Duhovny et al., 2005; Trott and Olsen, 2010). Before the molecular docking, all analysed molecular structures were optimised by additional software, such as the AutoDockTools software (Morris et al., 2009) for ADVina, or appropriate built-in modules to add charges, assign hydrogen atoms, and set up rotatable bonds. For the ADVina and PatchDock packages, the nanoparticle minimisation was done in a vacuum using semiempirical methods and the AM1 Hamiltonian as implemented in the Spartan software 10 v1.1.0 (Wavefunction, Inc. Irvine, CA).

ADVina rigid-flexible molecular docking uses a sophisticated gradient optimisation algorithm in its local optimisation procedure, which was performed to the center of binding cavity using Cartesian coordinates: $\mathrm{x}=19.11 \AA, \mathrm{y}=52.37 \AA$, and $\mathrm{z}=-0.30 \AA$. The docking grid with a dimension of $30 \AA$ x $30 \AA$ x $30 \AA$ was used in each docking calculation with an exhaustiveness option of 8 (average accuracy). The ADVina output results represented the docking scores as Gibbs free energy of binding $(\Delta G)$ and were further converted to the predicted inhibition constants $\left(\mathrm{K}_{\mathrm{i}}\right)$. $\mathrm{K}_{\mathrm{i}}$ values for all docked poses were calculated from the $\Delta \mathrm{G}$ values as follows:

$$
\begin{aligned}
& \Delta G=-R T\left(\ln K_{i}\right), \\
& K_{i}=e^{\frac{-\Delta G}{R T}},
\end{aligned}
$$

where $\mathrm{R}$ (gas constant) is $1.98 \mathrm{cal}^{*}\left(\mathrm{~mol}^{*} \mathrm{~K}\right)^{-1}$, and $\mathrm{T}$ (room temperature) is $298.15 \mathrm{~K}$.

For FastDock experiment, the minimisation was performed by the built-in engine, which operated through a Lamarckian genetic algorithm, so that analysed carbon nanoparticles adapted energetically to the surrounding environment (Yadav et al., 2010). The best fits were sustained through analysing the potential of mean force (PMF) scores of each chromosome and assigning more reproductive opportunities to the chromosomes having lower scores (Meugge et al., 1999; Yadav et al., 2010). This process was repeated for almost 3000 generations with 500 individuals and 100,000 energy evaluations using the Fast-Dock-Manager and Fast-Dock-Compute engines available within the ProjectLeader module of Scigress Explorer. Other parameters of genetic algorithm, such as crossover rate, elitism, mutation rate, and convergence, were left to their default values according to the experimental setup taken from the literature (Yadav et al., 2010). PatchDock employed shape-based rigid-rigid docking, devised from complementarity principles, and spherical harmonics to assess geometric feature-based shape matching. The PatchDock program is built on the basis of a rigid-body geometric hashing algorithm, dividing molecular surfaces into patches according to the molecular shape and comparing these patches to generate a set of candidate transformations (Duhovny et al., 2002; Benyamini et al., 2006). Each transformation was ranked by geometric complementarity score as PatchDock score (PDS) and atomic contact energy (ACE); the last parameter estimates atomic dissolvation energy of the obtained complex (Zhang et al., 1997; Duhovny et al., 2002). Through the docking process, redundant solutions were discarded by $1.5 \AA$ RMSD (root-mean-square-deviation) clustering. Physicochemical properties, such as molecular surface area for analysed nanostructures were calculated with the DS ViewerPro 6.0 (Accelrys, Inc., San Diego, CA). Molecular 
graphics and visualisation were performed with the PyMol software (DeLano; 2002). The hexagon/pentagon $\left(\mathrm{A}_{h} / \mathrm{A}_{\mathrm{p}}\right)$, total $\left(\mathrm{A}_{\text {total }}\right), \pi-\pi$ stacking $\left(\mathrm{A}_{\pi-\pi}\right)$, and residual $\left(\mathrm{A}_{\text {res }}\right)$ areas were calculated using following equations:

$$
\begin{aligned}
& A_{h}=2.59 t^{2}, \\
& A_{p}=1.72 t^{2}, \\
& A_{\text {total }}=A_{h(\text { total })}^{l} n+A_{p(\text { total })}^{l} \\
& A_{\pi-\pi}=A_{h(\pi-\pi)}^{r}+A_{h(\pi-\pi)}^{l}+A_{p(\pi-\pi)}^{l}, \\
& A_{\text {res }}=A_{\text {total }}-A_{\pi-\pi},
\end{aligned}
$$

where $t$ is the side length in $\AA ; A_{h(\text { total })}^{l}$ and $A_{p(\text { total })}^{l}$ are a total number of ligand hexagon and pentagon areas, $A_{h(\pi-\pi)}^{r}, A_{h(\pi-\pi)}^{l}$, and $A_{p(\pi-\pi)}^{l}$ are a number of receptor hexagon areas and ligand hexagon and/or pentagon areas involved in $\pi$ - $\pi$ stacking. Statistical linear regression analyses and graphical representations were performed with GraphPad Prism v.4 for Windows (GraphPad Software, San Diego, CS). The differences were considered statistically significant at $p<0.0007$.

\section{Results and discussion}

\subsection{Analysis of ADVina docking results}

We initially developed a docking strategy to the P-gp protein using a number of carbon allotropes that were not yet confirmed to be well-known P-gp substrates or inhibitors.

As a first step, we docked buckminsterfullerenes $\left(\mathrm{C}_{20}-\mathrm{C}_{70}\right)$ and single-wall carbon nanotube to the ligand binding site associated with the QZ59-RRR molecule of murine Pgp protein by measuring binding affinities. Notably, the human and murine P-gp homologues showed a high amount of conservation with $87 \%$ of sequence identity and $\sim 100 \%$ identity within the binding site cavity between these two species (Dolghih et al., 2011).

The structure of P-gp represents a nucleotide-free inward-facing conformation positioned as two "halves" where QZ59-RRR binds one site per transporter located in the center of the molecule between transmembrane domains (TM) 6 and 12. The inwardfacing conformation is believed to be competent for ligand binding at an initial stage of the efflux cycle (Aller et al., 2009).

Subsequently, a standard rigid-flexible ADVina molecular docking procedure yielded two main outcomes: conformational sampling (docking pose) of a ligand molecule within the receptor binding site and a score approximation function $(\Delta G)$ indicating the receptor-ligand interaction strength. Considering the crystal lattice rigidity of nanoparticles as a result of $\mathrm{sp} 2$ carbon hybridisation, the docking flexibility allowed them very limited lattice relaxation to establish the conformational change.

The top nine docking poses were visually inspected and emphasised by $\Delta \mathrm{G}$ and $\mathrm{K}_{\mathrm{i}}$ values to rank the different conformations of the receptor-ligand complex. The fullerene nanoparticles were docked into the $\mathrm{P}$-gp binding cavity with minimal $\Delta \mathrm{G}$ values of $-54.81 \mathrm{~kJ}^{*} \mathrm{~mol}^{-1}$ for $\mathrm{C}_{60}$ and $-56.90 \mathrm{~kJ}^{*} \mathrm{~mol}^{-1}$ for $\mathrm{C}_{70}$, respectively. The $\mathrm{C}_{20}$ molecule together with the QZ59-RRR docking pose showed a decrease in binding affinity $(-40.59$ 
$\mathrm{kJ}^{*} \mathrm{~mol}^{-1}$ for QZ59-RRR and $-32.22 \mathrm{~kJ}^{*} \mathrm{~mol}^{-1}$ for $\mathrm{C}_{20}$ ). The $\mathrm{C}_{168}$ (nanocapsule) docking was detected as energetically unfavorable for docking; it produced a positive $\Delta \mathrm{G}$ values from 0.0 (best pose) to $45.86 \mathrm{~kJ}^{*} \mathrm{~mol}^{-1}$ (worst pose) indicating either extremely low or complete absence of binding affinity for this type of nanoparticle. The $K_{i}$ values for all the docked poses were directly proportional to the corresponding $\Delta \mathrm{G}$ parameters (Figure 1(A)).

Figure 1 Distribution of different docking scores $(\Delta G, P M F)$ and binding affinities $\left(K_{i}\right)$ calculated using the ADVina (A) and FastDock $(\mathrm{B}, \mathrm{C})$ programs
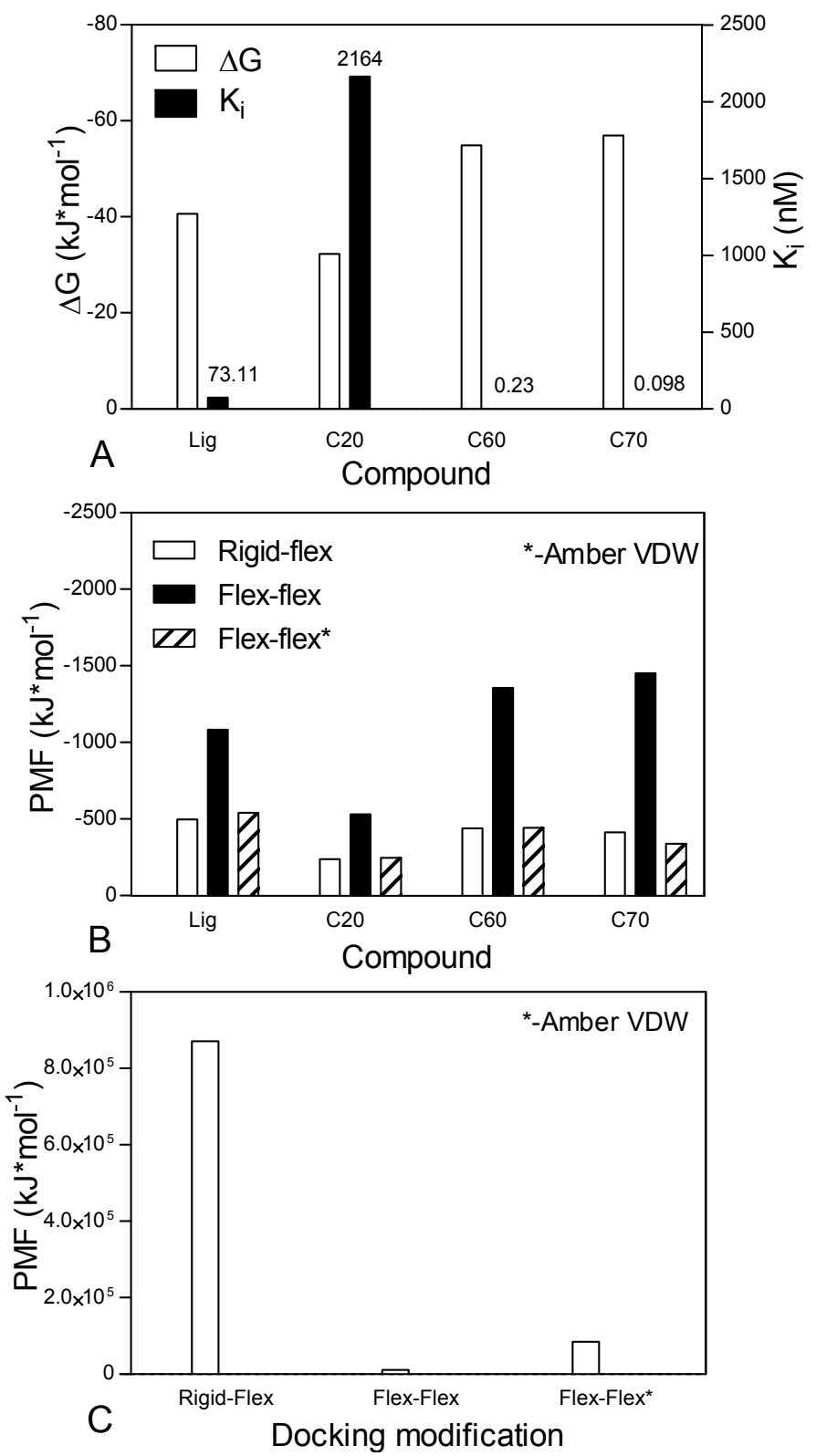


\subsection{Analysis of FastDock docking results}

The FastDock module in the Scigress software automates the docking of the nanoparticles into P-gp active site by using a genetic algorithm with a fast, simplified potential of mean force scoring function (Muegge and Martin, 1999). The PMF function is a knowledge-based approach that extracts pairwise atomic potentials from structure information of the receptor-ligand complexes, which are accumulated in the Protein Data Bank. PMF has been demonstrated to show a significant correlation between experimental binding affinities and its computed score for diverse holo-structures (Muegge et al., 1999; Ha et al., 2000; Muegge and Rarey, 2001). PMF uses atom types, which are similar to the empirical force-fields used in mechanics and dynamics simulations. Structure-based screening involves docking of candidate ligands into protein targets, followed by applying a PMF scoring function to estimate the likelihood that ligand will bind to the receptor molecule with low or high affinity (Muegge and Martin, 1999). Thus, three different docking modifications were performed to specify flexibility for the P-gp residues (binding site): rigid receptor to flexible ligand, flexible receptor to flexible ligand, and flexible receptor to flexible ligand with Amber van der Waals potentials.

Prior to molecular docking, we extracted the binding site from the P-gp molecule using $3 \AA$ threshold distance from the QZ59-RRR ligand. The extracted binding site consisted of predominantly aromatic amino acid residues, such as aromatic (Tyr303, Phe332, Phe339, Phe724, Tyr949, Phe974, Phe975), hydrophobic (Leu335, Ile336, Val978, Ala981) and hydrophilic (Gln721, Ser725, Ser975).

The best outcome was produced by flexible-flexible molecular docking with minimal PMF values of $-531 \mathrm{~kJ}^{*} \mathrm{~mol}^{-1}$ for $\mathrm{C}_{20},-1355.60 \mathrm{~kJ}^{*} \mathrm{~mol}^{-1}$ for $\mathrm{C}_{60}$, and $-1451.80 \mathrm{~kJ}^{*} \mathrm{~mol}^{-1}$ for $\mathrm{C}_{70}$ (Figure 1(B)). The QZ59-RRR-P-gp pose was used as a control with PMF of $-1083 \mathrm{~kJ}^{*} \mathrm{~mol}^{-1}$. The $\mathrm{C}_{168}$ docking run produced extremely high PMF values either for rigid-flexible or flexible-flexible docking (Figure 1(C)). Surprisingly, the energetic pattern produced by flexible-flexible docking was reminiscent of the $\Delta \mathrm{G}$ profile confirming similar binding affinities reproduced by different molecular docking programs. Expectedly, the rigid-flexible docking and flexible-flexible docking with optimised Amber van der Waals (VDW) potentials were characterised by higher PMF levels. This outcome could be explained either by the absence of total flexibility or by the fact that the standard PMF used specific 6-12 Lennard-Jones potentials for each pairwise interaction. These potentials permit hydrogen bonding and electrostatic interactions, which are sometimes prohibited by the VDW potentials.

\subsection{Analysis of PatchDock docking results}

The PatchDock experiment was designed in a way that no apriori additional data was used as an input for the docking server, such as Cartesian coordinates of receptor/ligand binding sites and distance constraints. Therefore, all possible surface pockets on the receptor molecule were fully detected automatically to "screen" the whole big P-gp protein, which is $136 \AA$ in length, $70 \AA$ in width, and with a molecular weight of $170 \mathrm{kDa}$.

It is well known that different docking methods are not always accurate enough to produce near-native conformations at the top and output many outcomes. That is why top ten solutions were manually inspected to emphasise the common clustering pattern. 
The output of PatchDock was a list of candidate complexes specified receptor and nanoparticle clearly showed the highest arbitrary score value for $\mathrm{C}_{168}$ molecule (8932) with minimal ACE value of $-3367.62 \mathrm{~kJ}^{*} \mathrm{~mol}^{-1}$. On the contrary to the previous docking results, the PatchDock outputs displayed that the PDS and ACE distribution rate depended on the molecular surface area (MSA), and that high values of MSA corresponded to minimal ACE levels (Figure 2 (A, B)). Hence, these poses obtained by rigid-rigid PatchDock approach appeared to be with more favorable negative docking scores. The analysed nanoparticles were docked outside the binding crevice where the co-crystallised QZ59-RRR ligand was located with different distances from its center (Figure 3): $60.1 \AA$ for $\mathrm{C}_{20}, 7.3 \AA$ for $\mathrm{C}_{168}, 4.3 \AA$ for $\mathrm{C}_{70}$, and $4.0 \AA$ for $\mathrm{C}_{60}$. The corresponding distances were measured from the center of the P-gp binding site to the center of the carbon allotrope molecule (COM).

Figure 2 Reported scoring functions for PatchDock (A). Data smoothing was applied to produce a fitting curve for the dynamical comparison (B)
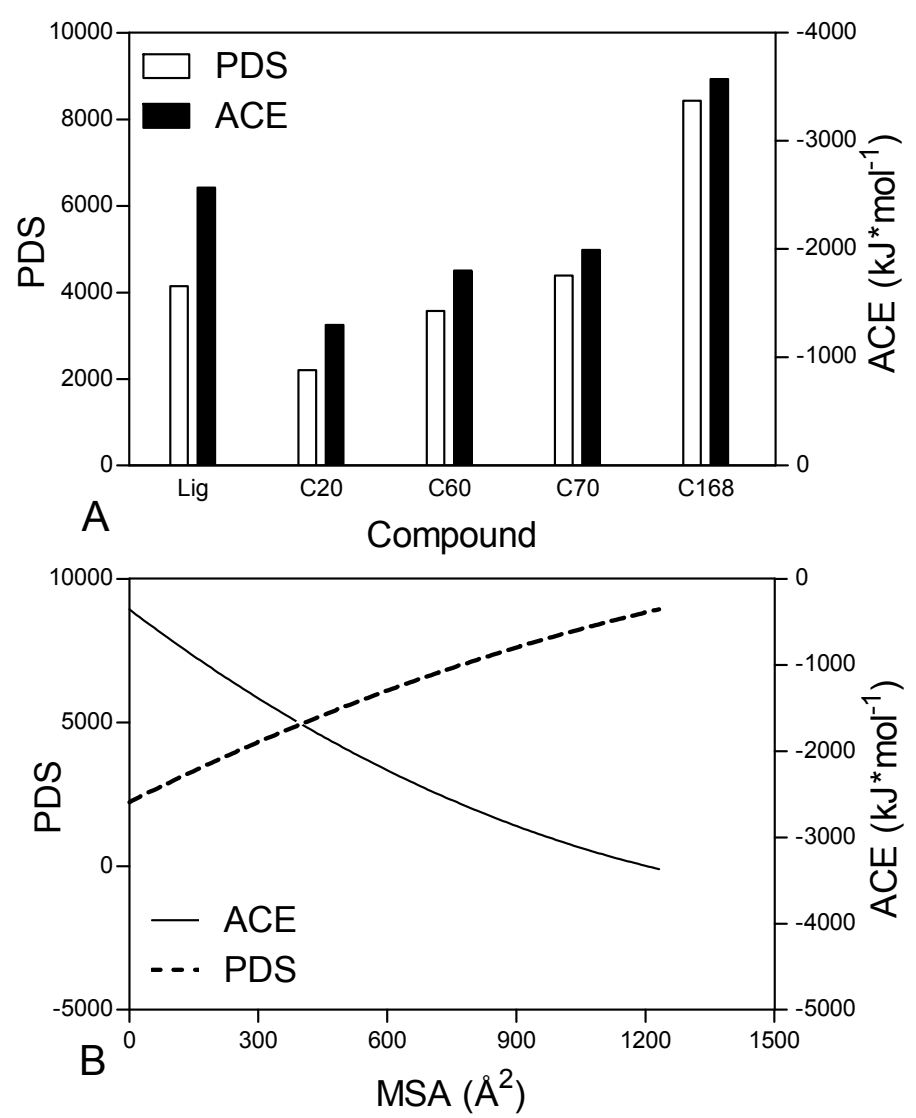

The $\mathrm{C}_{60}, \mathrm{C}_{70}$ and $\mathrm{C}_{168}$ molecules were distributed at the intracellular side, near pore entrance, with a slight shift from the QZ59-RRR, in proximity to the P-gp binding site. $\mathrm{C}_{20}$ occupied the "lower" site surrounded by carboxyl groups of hydrophilic (Asp1015, Gln1103, Arg1106) and hydrophobic (Ile1014) residues on nucleotide binding domain (NBD2). The $\mathrm{C}_{20}$ dislocation might be explained by either the small size of the $\mathrm{C}_{20}$ 
molecule $(3.9 \AA)$ or the fact that some hydrophobic residues located at NBD2 could act as fingers to capture the small molecule; thus prevent its translation into the P-gp arch portal formed by TMs 4 and 6 in order to permeate drug molecule from the lipid bilayer. In fact, the presence of multiple binding sites of P-gp, had already been proposed more than a decade ago, such as the H-site for Hoechst 33342 and the R-site for Rho 123 (Shapiro and Ling; 1997).

Figure 3 Docking patterns from top ten PatchDock outputs. The hydrophilic, hydrophobic, and aromatic residues are colored blue, green and magenta, respectively. Transmembrane (TMs) and nucleotide binding domains (NBDs) are also labelled (see online version for colours)

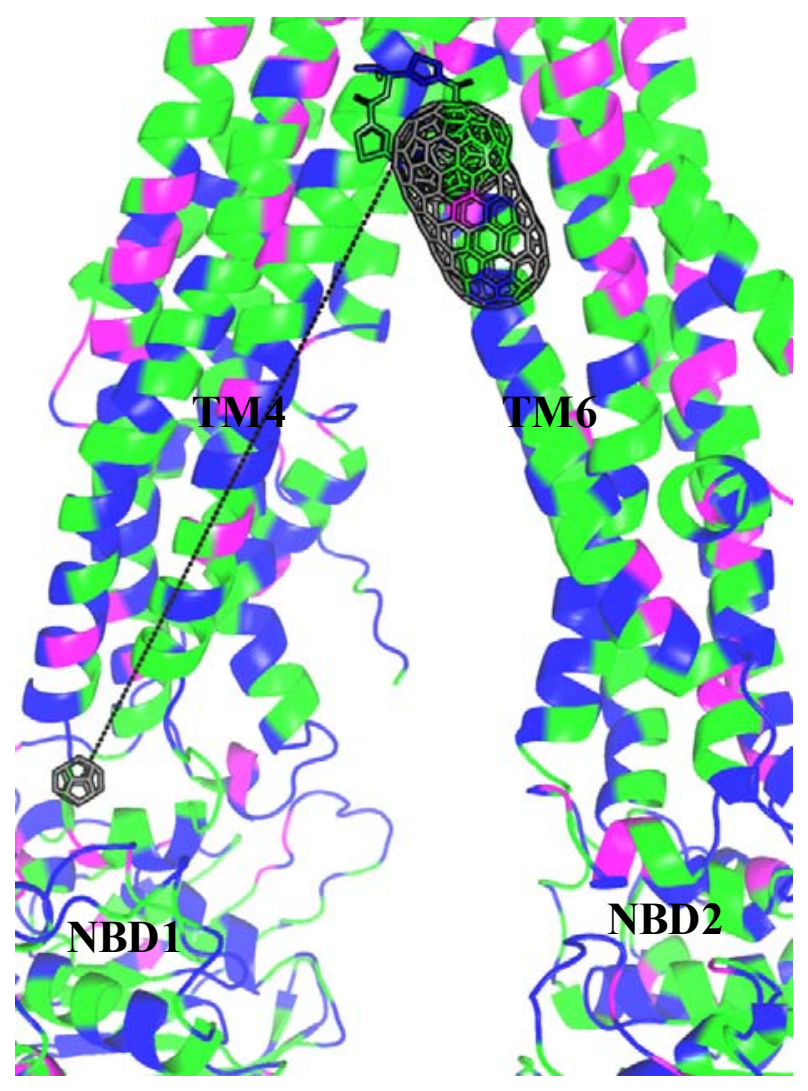

\subsection{Geometric analysis of $\pi$ - $\pi$ stacking effects}

The mechanisms of protein-nanoparticle interaction include different noncovalent interactions such as dipolar, ionic interactions, hydrogen bonding, van der Waals interactions, etc. Moreover, the $\pi-\pi$ stacking effect plays a nontrivial role in carbon nanoparticle interactions, such as fullerenes and carbon nanotubes referring to attractive forces between aromatic rings (Miller et al., 2000; Tournus et al., 2005; Sygula et al., 2007; Roquelet et al., 2010). Manual inspections of docking poses at the interface between P-gp and nanoparticles have revealed a domination of the hydrophobic 
interactions and $\pi-\pi$ stacking effects as a predominant recognition mode. Traditionally, the $\pi-\pi$ stacking evaluation depends heavily on the quantum mechanical calculation of quadrupole moments for the interacting species (Luhmer et al., 1994). At this point, the geometric analysis of $\pi-\pi$ stacking effects would be rational, computationally simple, and relatively accurate without the time consuming techniques.

In order to explain the energetic profiles caused by the gradient optimisation method, it was set out to analyse the ADVina docking profiles of nanoparticles and P-gp using geometric configuration of aromatic rings involved in supramolecular $\pi-\pi$ stacking. To achieve this task, the distances were estimated from the aromatic ring center of P-gp residues involved in the $\pi-\pi$ stacking to the closest ring center in the nanoparticle lattice ( $\pi-\pi$ stacking distances) or to COM (Figure 4).

Figure 4 Geometric configuration of $\pi-\pi$ stacking; see text for details (see online version for colours)

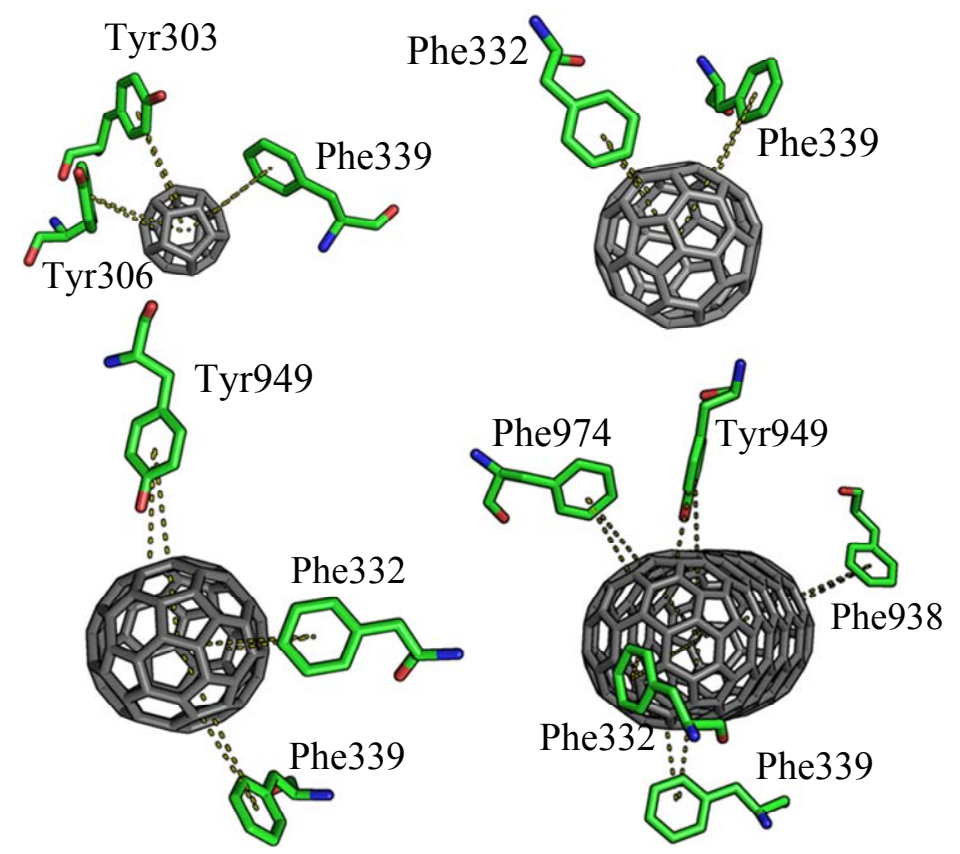

Despite the fact that $\mathrm{C}_{168}$ is not fit to the P-gp binding site concerning excessively high $\Delta \mathrm{G}$ and PMF values, its $\pi-\pi$ stacking mode comparison to the rest of the nanostructures might elucidate these energetic discrepancies. Ideally, tyrosine and phenylalanine residues interact with adjacent parts of nanoparticles by their aromatic rings in sandwich, t-shape, or parallel-displaced manner. In reality, the aromatic rings are subjected to a different tilting of their planes coursed by rotational effect as shown in Figure 4 . The $\pi-\pi$ stacking distances were the same in all nanoparticles whereas distance elevation from $\mathrm{COM}$ was observed due to $\mathrm{C}_{20} / \mathrm{C}_{60}$ diameter and $\mathrm{C}_{70} / \mathrm{SWCNT}$ length range increase (Figure 5 (A, B)). Also, the prevalence of the $\pi-\pi$ stacking effect at the poles of SWCNT also contributed to the previous observations. 
Figure 5 Reported $\pi-\pi$ stacking distances alone and in conjunction with molecular surface area (MSA) for different nanoparticles (A). Calculated $\mathrm{A}_{\text {total }}$ and $\mathrm{A}_{\text {res }}$ values are characterised by using the linear regression analysis to determine the relationship strength (B)
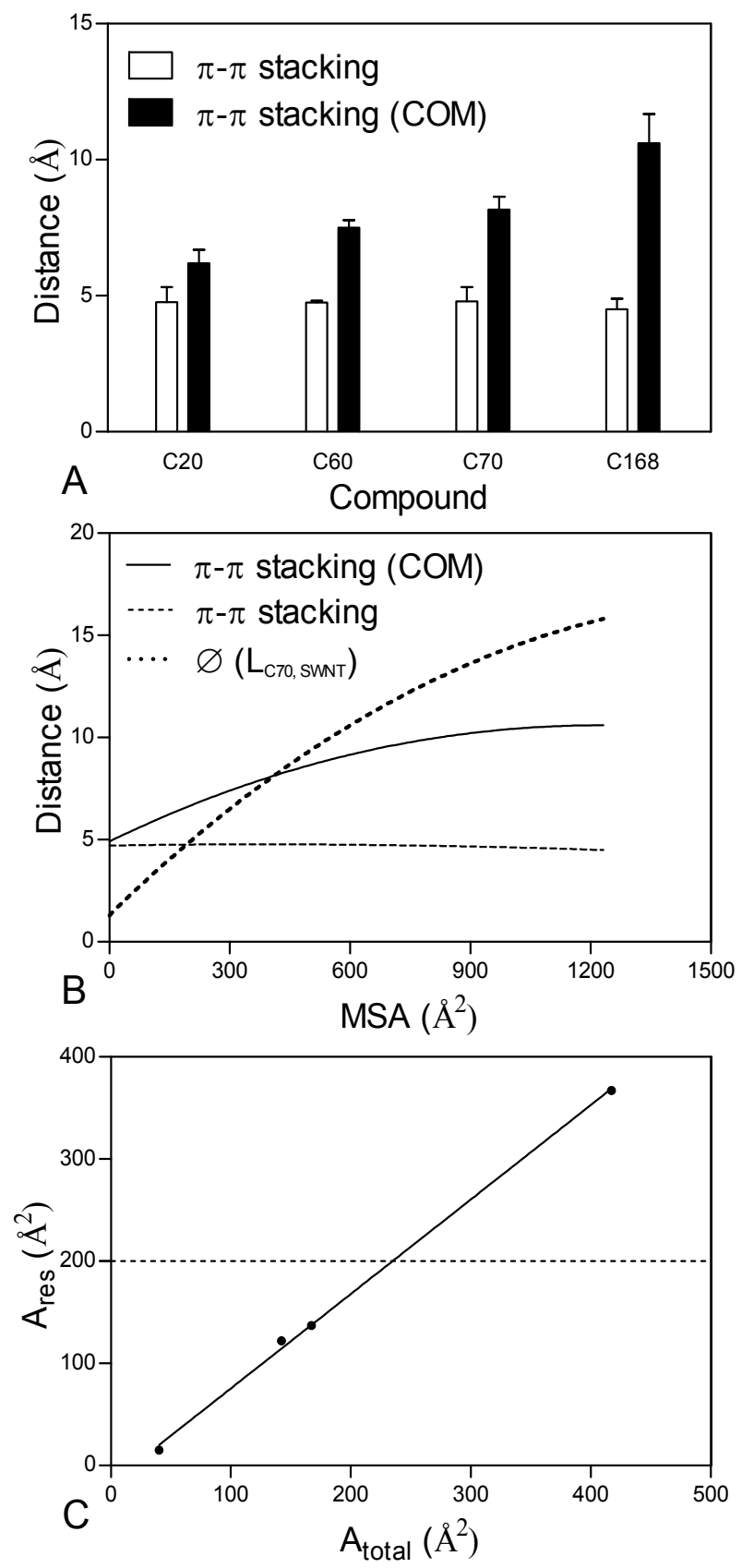
To calculate the residual area that is not involved in the $\pi-\pi$ stacking, it was taken into account that the overall amount of hexagons and pentagons in carbon lattice were the total area and area of $\pi-\pi$ contacts between receptor and nanostructure. At this point, the $\mathrm{C}_{20}$ molecule with dodecahedral cage structure is the smallest member of the fullerene family with $\mathrm{C}_{5}-\mathrm{C}_{5}$ bonds forming only 12 pentagons. For this molecule, one could also expect that the pentagonal lattice itself might alter the energetic affinity via $\pi$ - $\pi$ stacking mismatch despite the relatively higher number of P-gp interacting residues (Figure 4).

The $\mathrm{C}_{60}$ buckyball and $\mathrm{C}_{70}$ molecule, which is related to the truncated icosahedron, together with SWCNT form an additional 20, 25, and 74 hexagons with $\mathrm{C}_{5}-\mathrm{C}_{6}$ bonds to already existing 12 pentagons. Consequently, the obtained $\mathrm{A}_{\text {total }}$ and $\mathrm{A}_{\mathrm{res}}$ values represented relationship of correlation by a linear regression analysis with high Pearson's coefficient $\left(\mathrm{r}^{2}=0.99\right.$ ), and $\mathrm{P}<0.0007$ (Figure $5(\mathrm{C})$ ). The distribution rate occurred in a manner that high levels of $\Delta \mathrm{G}\left(\mathrm{K}_{\mathrm{i}}\right)$ and PMF correlate with high levels of the $\mathrm{A}_{\text {res }}$ parameters because of shape increase and elevation of the MSA/ $\mathrm{A}_{\text {total }}$ values.

\section{Conclusion}

The results of theoretical methods were presented to measure the recognition of carbon nanoparticles by P-gp, hypothesising that buckyballs appeared to be able to bind with P$\mathrm{gp}$ and might further be removed from the cell interior while SWCNT might not because of unfavourable interaction with the protein molecule. Interestingly, the results actually countered with the previous experimental findings (Xu et al., 2011) supporting the hypothesis that some carbon nanoparticles $\left(\mathrm{C}_{60}\right)$ are probably not the P-gp binders $(\mathrm{Xu}$ et al., 2012). However, this idea is mainly based on the solitary and implicit experimental results rather than on the well-confirmed data from different research groups. To probe this hypothesis, different outcomes were created which demonstrated the docking studies that some representative nanostructures, such as buckyballs $\left(\mathrm{C}_{20}, \mathrm{C}_{60}, \mathrm{C}_{70}\right)$ could be bound to the P-gp molecule, indicating the possibility of being competitive substrates of P-gp. As a possible outcome of this interaction, these molecules might be expelled from the target cells by judging their low levels of $\Delta \mathrm{G}\left(\mathrm{K}_{\mathrm{i}}\right)$ and PMF values. In contrast to fullerenes, the armchair SWCNT (nanocapsule) might not be efficiently effluxed by this type of the $\mathrm{ABC}$ transporter because of very high $\Delta \mathrm{G}$ which indicated extremely low binding affinity. Surprisingly, the shape-based docking approach (PatchDock) revealed that SWCNT maintained the highest shape congruence to P-gp according to low ACE parameters and increased nanoparticle propensity, especially for $\mathrm{C}_{20}$ to interact outside the QZ59-RRR-associated binding pocket. The interpretation for this important finding might be explained by insufficient shape similarities between the binding site and analysed nanostructures or by the presence of multiple binding sites of P-gp.

In addition, it is found for the first time that the $\pi-\pi$ stacking effect has contributed significantly with a total decrease in binding affinity by the elevation of the $A_{\text {res }}$ values following by the distance elongation from COM. Until recently, the docking studies concerning the recognition between the non-modified carbon nanoparticles and P-gp have not been available. Thus, it is tempting to say that the information obtained from this analytical work is essential for the fullerene and nanotube-based development of advanced drug delivery vectors. 


\section{Acknowledgements}

Special thanks are extended to Anna Poon from the City College of New York for her assistance in paper writing. The authors are grateful to the IZKF (Interdisziplinäres Zentrum für Klinische Forschung der Universität Würzburg) and the BMBF (Bundesministerium für Bildung und Forschung) for the support of this work by providing grants (BMBF01, EO1004) to Carola Förster.

\section{References}

Alibert-Franco, S., Pradines, B. et al. (2009) 'Efflux mechanism, an attractive target to combat multidrug resistant Plasmodium falciparum and Pseudomonas aeruginosa', Curr Med Chem, Vol. 16, No. 3, pp.301-317.

Aller, S.G., Yu, J. et al. (2009) 'Structure of P-glycoprotein reveals a molecular basis for polyspecific drug binding', Science, Vol. 323, No. 5922, pp.1718-1722.

Benyamini, H., Shulman-Peleg, A. et al. (2006) 'Interaction of c(60)-fullerene and carboxyfullerene with proteins: docking and binding site alignment', Bioconjug Chem, Vol. 17, No. 2, pp.378-386.

Bonn, G.K., Bakry, R., Vallant, R.M., Najam-ul-Haq, M., Rainer, M., Szabo, Z. and Huck, C.W. (2007) 'Medicinal applications of fullerenes', International Journal of Nanomedicine, Vol. 2, No. 4, pp.639-649.

Cataldo, F. and Da Ros, T. (2008) Medicinal chemistry and pharmacological potential of fullerenes and carbon nanotubes, Springer Science.

Cheng, J., Meziani, M.J. et al. (2011) 'Poly(ethylene glycol)-conjugated multi-walled carbon nanotubes as an efficient drug carrier for overcoming multidrug resistance', Toxicol Appl Pharmacol, Vol. 250, No. 2, pp.184-193.

Chiche, L., Gregoret, L.M., Cohen, F.E. and Kollman, P.A. (1990) 'Protein model structure evaluation using the solvation free energy of folding', Proc Natl Acad Sci USA, Vol. 87, No. 8, pp.3240-3243.

DeLano, W.L. (2002) The PyMOL Molecular Graphics System, San Carlos, CA, DeLano Scientific.

Dolghih, E., Bryant, C. et al. (2011) 'Predicting binding to p-glycoprotein by flexible receptor docking', PLoS Comput Biol, Vol. 7, No. 6.

Duhovny, D., Nussinov, R. and Wolfson, H.J. (2002) 'Efficient Unbound Docking of Rigid Molecules', in Gusfield et al. (Eds): Proceedings of the 2'nd Workshop on Algorithms in Bioinformatics(WABI), Rome, Italy, pp.185-200.

Foley, S., Crowley, C. et al. (2002) 'Cellular localisation of a water-soluble fullerene derivative', Biochem Biophys Res Commun, Vol. 294, No. 1, pp.116-119.

Gottesman, M.M., Fojo, T. et al. (2002) 'Multidrug resistance in cancer: role of ATP-dependent transporters', Nat Rev Cancer, Vol. 2, No. 1, pp.48-58.

Ha, S., Andreani, R., Robbins, A. and Meugge, I. (2000) 'Evaluation of docking/scoring approaches: A comparative study based on MMP3 inhibitors', J. Comp.-Aided Mol. Design, Vol. 14, pp.435-448.

Johnston, H.J., Hutchison, G.R., Christensen, F.M., Aschberger, K. and Stone, V. (2010) 'The biological mechanisms and physicochemical characteristics responsible for driving fullerene toxicity', Toxicol. Sci., Vol. 114, No. 2, pp.162-182.

Kam, N.W., Liu, Z. et al. (2005) 'Functionalization of carbon nanotubes via cleavable disulfide bonds for efficient intracellular delivery of siRNA and potent gene silencing', $J$ Am Chem Soc, Vol. 127, No. 36, pp.12492-12493. 
Koizumi, S., Konishi, M. et al. (1995) 'Flow cytometric functional analysis of multidrug resistance by Fluo-3: a comparison with rhodamine-123', Eur J Cancer, Vol. 31A, No. 10, pp.1682-1688.

Li, R., Wu, R. et al. (2010) 'P-glycoprotein antibody functionalized carbon nanotube overcomes the multidrug resistance of human leukemia cells', ACS Nano, Vol. 4, No. 3, pp.1399-1408.

Liu, Z., Winters, M. et al. (2007) 'siRNA delivery into human T cells and primary cells with carbon-nanotube transporters', Angew Chem Int Ed Engl, Vol. 46, No. 12, pp.2023-2027.

Luhmer, M., Bartik, K., Dejaegere, A., Bovy, P. and Reisse, J. (1994) 'The Importance of Quadrupolar Interactions in Molecular Recognition processes Involving a Phenyl Group', Bull. Soc. Chim. Fr., Vol. 131, pp.603-606.

Mazel, M., Clair, P. et al. (2001) 'Doxorubicin-peptide conjugates overcome multidrug resistance', Anticancer Drugs, Vol. 12, No. 2, pp.107-116.

Meugge, I., Martin, Y., Hajduk, P.J. and Fesik, S.W. (1999) 'Evaluation of PMF Scoring in Docking Weak Ligands to the FK506 Binding Protein', J. Med. Chem., Vol. 42, pp.2498-2503.

Miller, G.P., Mack, J. et al. (2000) 'pi-stacking interactions in cis-Bisfullerene', Org Lett, Vol. 2, No. 25, pp.3983-3986.

Morris, A.L., MacArthur, M.W., Hutchinson, E.G. and Thornton, J.M. (1992) 'Stereochemical quality of protein structure coordinates', Proteins, Vol. 12, No. 4, pp.345-364.

Morris, G.M., Huey, R. et al. (2009) 'AutoDock4 and AutoDockTools4: Automated docking with selective receptor flexibility', J Comput Chem, Vol. 30, No. 16, pp.2785-2791.

Muegge, I. and Martin, Y. (1999) 'A General and Fast Scoring Function for Protein-Ligand Interactions: A Simplified Potential Approach', J. Med. Chem., Vol. 42, pp.791-804.

Muegge, I. and Rarey, M. (2001) 'Small Molecule Docking and Scoring', Rev. in Comp. Chem., Vol. 17, pp.1-60.

Pantarotto, D., Briand, J.P. et al. (2004) 'Translocation of bioactive peptides across cell membranes by carbon nanotubes', Chem Commun (Camb), Vol. 1, pp.16-17.

Pantarotto, D., Partidos, C.D. et al. (2003) 'Immunization with peptide-functionalized carbon nanotubes enhances virus-specific neutralizing antibody responses', Chem Biol, Vol. 10, No. 10, pp.961-966.

Pantarotto, D., Singh, R. et al. (2004) 'Functionalized carbon nanotubes for plasmid DNA gene delivery', Angew Chem Int Ed Engl, Vol. 43, No. 39, pp.5242-5246.

Porter, A.E., Muller, K. et al. (2006) 'Uptake of C60 by human monocyte macrophages, its localization and implications for toxicity: studied by high resolution electron microscopy and electron tomography', Acta Biomater, Vol. 2, No. 4, pp.409-419.

Roquelet, C., Lauret, J.S. et al. (2010) 'Pi-stacking functionalization of carbon nanotubes through micelle swelling', Chemphyschem, Vol. 11, No. 8, pp.1667-1672.

Sayes, C.M., Gobin, A.M. et al. (2005) 'Nano-C60 cytotoxicity is due to lipid peroxidation', Biomaterials, Vol. 26, No. 36, pp.7587-7595.

Schneidman-Duhovny, D., Inbar, Y. et al. (2005) 'PatchDock and SymmDock: servers for rigid and symmetric docking', Nucleic Acids Res 33(Web Server issue), pp. W363-Q367.

Shapiro, A.B. and Ling, V. (1997) 'Positively cooperative sites for drug transport by P-glycoprotein with distinct drug specificities', Eur J Biochem, Vol. 250, No. 1, pp.130-137.

Singh, R., Pantarotto, D. et al. (2005) 'Binding and condensation of plasmid DNA onto functionalized carbon nanotubes: toward the construction of nanotube-based gene delivery vectors', J Am Chem Soc, Vol. 127, No. 12, pp.4388-4396.

Sygula, A., Fronczek, F.R. et al. (2007) 'A double concave hydrocarbon buckycatcher', J Am Chem Soc, Vol. 129, No. 13, pp.3842-3843.

Tournus, F., Latil, S., Heggie, M.I. and Charlier, J.C. (2005) 'pi-stacking interaction between carbon nanotubes and organic molecule', Physical Review B, Vol. 72, No. 7, pp.1-5. 
Trott, O. and Olson, A.J. (2010) 'AutoDock Vina: improving the speed and accuracy of docking with a new scoring function, efficient optimization, and multithreading', J Comput Chem, Vol. 31, No. 2, pp.455-461.

Willard, L., Ranjan, A. et al. (2003) 'VADAR: a web server for quantitative evaluation of protein structure quality', Nucleic Acids Res, Vol. 31, No. 13, pp.3316-3319.

Xia, T., Li, N. and Nel, A.E. (2009) 'Potential health impact of nanoparticles', Annu. Rev. Public Health, Vol. 30, pp.137-150.

$\mathrm{Xu}, \mathrm{X}$., Li, R.B., et al. (2012) 'Multidrug resistance protein P-glycoprotein does not recognize nanoparticle C-60: experiment and modeling', Soft Matter, Vol. 8, No. 10, pp.2915-2923.

Yadav, D.K., Meena, A., Srivastava, A., Chanda, D., Khan, F. and Chattopadhyay, S.K. (2010) 'Development of QSAR model for immunomodulatory activity of natural coumarinolignoids', Drug Des Devel Ther, Vol. 4, pp.173-186.

Zhang, C., Vasmatzis, G., Cornette, J.L. and DeLisi, C. (1997) 'Determination of atomic desolvation energies from the structures of crystallized proteins', J Mol Biol., Vol. 267, No. 3, pp.707-726.

Zhang, J., Liang, Y. and Zhang, Y. (2011) 'Atomic-Level Protein Structure Refinement Using Fragment-Guided Molecular Dynamics Conformation Sampling', Structure, Vol. 19, pp.1784-1795. 